following diagnosis and 109 (19.2\%) following surgery. There were improved survival times following diagnosis for patients prescribed a statin with OC overall $(\mathrm{HR}=0.71,95 \% \mathrm{CI}=0.64-0.79, \mathrm{p}<0.001)$ and specifically $\mathrm{OAC}(\mathrm{HR}=0.58,95 \% \mathrm{CI}=0.43-0.78, \mathrm{p}<0.001)$. Furthermore, statin use post-oesophagectomy was associated with improved survival in those with OC overall ( $\mathrm{HR}=0.66,95 \%$ $\mathrm{CI}=0.48-0.91, \mathrm{p}=0.013)$ and those with OAC specifically $(\mathrm{HR}=0.58,95 \% \mathrm{CI}=0.30-1.10, \mathrm{p}=0.096)$ with borderline significance. There were no significant effects between statin use and survival in patients with OSCC. Associations between statin use and overall survival were very similar to their effect on disease-specific survival.

Conclusion Statin use in patients with OC either after diagnosis or post-oesophagectomy is associated with improved survival. This data suggests a need for randomised controlled trials of statins in patients with OC.

Disclosure of Interest None Declared

\section{OC-029 RADIOFREQUENCY ABLATION (RFA) CONFERS SUSTAINED BENEFIT FOR SOUAMOUS HIGH GRADE DYSPLASIA (HGD) AND EARLY SQUAMOUS CELL CARCINOMA (SCC) IN PATIENTS WHO DO NOT PROGRESS FOLLOWING FIRST TREATMENT}

doi:10.1136/gutjnl-2013-304907.029

${ }^{1,2, *} \mathrm{R}$ J Haidry, ${ }^{3} \mathrm{M}$ Banks, ${ }^{4} \mathrm{M}$ Butt, ${ }^{3} \mathrm{~A}$ Gupta, ${ }^{5} \mathrm{~J}$ Louis-Auguste, ${ }^{6} \mathrm{~J}$ Dunn, ${ }^{7} \mathrm{H} \mathrm{L}$ Smart, ${ }^{8 P}$ Bhandari, 9 L-A Smith, ${ }^{10} \mathrm{R}$ Willert, ${ }^{11} \mathrm{G}$ Fullarton, ${ }^{12} \mathrm{M}$ di Pietro, ${ }^{13}$ Penman, ${ }^{14} \mathrm{R}$ Narayanasamy, ${ }^{11} \mathrm{~J}$ Morris, ${ }^{14} \mathrm{D} 0$ 'Toole, ${ }^{3} \mathrm{M}$ Novelli, ${ }^{15} \mathrm{C}$ Gordon, ${ }^{3,4} \mathrm{~L}$ Lovat. ${ }^{1}$ National Medical laser Centre (NMLC), University College London (UCL), London; ${ }^{2}$ University College London Hospital (UCLH) NHS foundation Trust, Lonodn; ${ }^{3} U C L H ;{ }^{4} N M L C$, UCL, London; ${ }^{5} \mathrm{NMLC}$, UCL, Lonodn; ${ }^{6} G u y$ 's \& St Thomas' Hospitals, London; 'Royal Liverpool University Hospital, Liverpool; ${ }^{8}$ Princess Alexandra Hospital, Portsmouth; ${ }^{9}$ Bradford Teaching Hospital, Bradford; ${ }^{10} \mathrm{Central}$ Manchester University Hospital, Manchester; ${ }^{11} \mathrm{GRI}$, Glasgow; ${ }^{12}$ Adenbrookes Hospital, Cambridge; ${ }^{13}$ Royal Infirmary, Edinburgh, UK; ${ }^{14}$ St James Hospital, Dublin, Ireland; ${ }^{15}$ Royal Bournemouth Hospital, Bournemouth, UK

Introduction Oesphageal SCC carries a poor prognosis. Squamous HGD is the precursor lesion to SCC. Risk of progression to SCC with HGD can be $65 \%$ at 5 years. RFA is a minimally invasive technique with proven efficacy for early neoplasia arising in Barrett's oesophagus. We present prospective data from 10 centres in the United Kingdom (UK) HALO registry.

Methods Superficial lesions were removed by endoscopic mucosal resection (EMR) before RFA. Treatment consisted of a single ablation at $12 \mathrm{~J} / \mathrm{cm} 2$. Patients were followed up 3 months after treatment with biopsies. Those with residual dysplasia underwent further RFA until 12 months when they were assessed for treatment success or failure. Recurrent dysplasia was retreated with EMR/RFA. Primary outcomes were reversal of dysplasia (CR-D) at 12 months.

Results 26 patients had RFA. Mean length mucosa ablated was 5.3 $\mathrm{cm}$ (1-14). 7/26 (27\%) had EMR before RFA. Prior EMR did not confer benefit to outcome, nor did baseline disease length. Following first RFA, $6 / 26$ patients (23\%) progressed to invasive disease. Only one more patient progressed later in treatment course. CR-D was achieved in $50 \%$ at protocol end, mean 1.7 RFA treatments (1-4). 10/13 (77\%) with successful RFA at 12 months remain disease free at most recent follow up (median 21 months). Kaplan Meier statistics show 2 years post treatment $68 \%$ patients are likely to remain in remission from dysplasia for those with successful outcome at 12 months. 5 patients (19\%) required dilatations for oesophageal stricturing.

Conclusion Squamous HGD \& CIS are aggressive pathologies as evidenced by the fact that $23 \%$ patients in our cohort progressed to invasive disease despite RFA. However the majority who do not progress early (13/19 patients) achieve benefit \& are more likely to have a successful \& durable outcome. There is limited experience in the UK with RFA in these patients. Pre RFA EMR for visible lesions is limited in our series. As a result some patients may be under staged prior to RFA which may account for the high rate of progression after first treatment.

Disclosure of Interest None Declared

\section{OC-030 BARRETT'S EPITHELIUM SHOWS EVIDENCE OF GASTRIC AND INTESTINAL DIFFERENTIATION PROGRAMMES BUT PRESERVES THE PROLIFERATIVE AND STEM CELL ARCHITECTURE OF GASTRIC GLANDS}

doi:10.1136/gutjnl-2013-304907.030

${ }^{1} \mathrm{D}$ Lavery, ${ }^{2} \mathrm{~A}$ Nicholson, ${ }^{3} \mathrm{R}$ Jeffery, ${ }^{3} \mathrm{R}$ Poulsom, ${ }^{4} \mathrm{H}$ Barr, ${ }^{3} \mathrm{~J}$ Jankowski, ${ }^{5} \mathrm{M}$ Novelli, ${ }^{6} \mathrm{~N}$ Shepherd, ${ }^{5} \mathrm{M}$ Rodriguez-Justo, ${ }^{7} \mathrm{M}$ Jansen, ${ }^{1} \mathrm{~N}$ A Wright, ${ }^{1,} \mathrm{~S}$ A C Mcdonald. ${ }^{1}$ Centre for Tumour Biology, Barts and the London School of Medicine and Dentistry, London; ${ }^{2}$ Winton Group, Cancer Research UK Cambridge Institute, Cambridge; ${ }^{3}$ Centre for Digestive Diseases, Barts and the London School of Medicine and Dentistry, London; ${ }^{4}$ Department of Surgery, Gloucestershire Hospitals NHS Trust, Gloucester; ${ }^{5}$ Department of Pathology, University College London Hospitals, London; ${ }^{6}$ Gloucestershire Cellular Pathology Laboratory, Cheltenham General Hospital, Cheltenham, UK; 'Department of Pathology, Academic Medical Center, Amsterdam, Netherlands

Introduction The origin and development of Barrett's oesophagus has long been discussed, with three main proposals for the origin of metaplasia: from oesophageal squamous epithelium, from upward migration of cardiac glands, or from submucosal glands.

Methods In Barrett's glands we have studied the distribution of proliferative activity using Ki67 and the migration of cells in Barrett's glands from patients infused with iododeoxyuridine 7 and 11 days pre-oesophagectomy. We have localised gene expression of mucins using immunohistochemistry (IHC) and trefoil family factor (TFF) peptides using IHC and in situ hybridization (ISH) and the stem cell marker Lgr5 using ISH, combining this with analysis of the clonal architecture of Barrett's glands using mitochondrial DNA (mtDNA) as a clonal marker.

Results In Barrett's glands proliferation is seen mainly in the middle part of the gland and diminishes towards the surface and the base of the gland. Cells migrate in a bidirectional manner. MUC5AC and TFF1 expression are found superficially, while MUC6 and TFF2 are found at the bases of the glands, similar to the distribution seen in antral gastric glands: MUC2, staining goblet cells and columnar cells, is concentrated superficially. Lgr 5 mRNA is also found in the middle part of the glands, indicating the location of the stem cell niche. Barrett's glands are clonal, indicating derivation from a single cell, and suggesting that Barrett's stem cells have dual differentiation capacity. Gastric intestinal metaplasia, on the other hand, shows basal Lgr5 mRNA localisation with a distribution of proliferative activity similar to intestinal crypts.

Conclusion We conclude that Barrett's glands show the proliferative and stem cell architecture, and preserve patterns of gene expression of pyloric-type gastric glands, but are maintained by unique stem cells with both gastric and intestinal differentiation capacity: we propose that Barrett's glands originate as gastric glands and that subsequent intestinal differentiation advances with time, strongly supporting an origin from the proximal cardiac mucosa.

Disclosure of Interest None Declared

OC-031 USING GENOME-WIDE STUDIES TO IDENTIFY NOVEL
FOXM1 TRANSCRIPTION FACTOR TARGET GENES IN
OESOPHAGEAL CANCER

doi:10.1136/gutjnl-2013-304907.031

$1,2{ }^{*} \mathrm{E}$ F Wiseman, ${ }^{3} \mathrm{~N}$ Han, ${ }^{1,2}, \mathrm{Y}$ S Ang, ${ }^{3} \mathrm{~A}$ S Sharrocks. ${ }^{1}$ Faculty of Medical and Human Sciences, University of Manchester, Manchester; ${ }^{2}$ Gastroenterology, Royal Albert Edward Infirmary, Wigan; ${ }^{3}$ Faculty of Life Sciences, University of Manchester, Manchester, UK 